\title{
Lower platelet counts were associated with 90-day adverse outcomes in acute-on-chronic liver disease patients
}

\author{
Renjie Ouyang ${ }^{1,2 \#}$, Hai $\mathrm{Li}^{3 \#}$, Jie Xia ${ }^{4 \#}$, Xianbo Wang ${ }^{5 \#}$, Xin Zheng ${ }^{6 \#}$, Yan Huang ${ }^{7 \#}$, Zhongji Meng $^{8}$, \\ Yanhang Gao ${ }^{9}$, Zhiping Qian ${ }^{10}$, Feng Liu ${ }^{11}$, Xiaobo Lu ${ }^{12}$, Yu Shi ${ }^{13}$, Jia Shang ${ }^{14}$, Junping Liu ${ }^{14}$, \\ Guohong Deng ${ }^{4}$, Yubao Zheng ${ }^{15}$, Huadong Yan ${ }^{16}$, Weituo Zhang ${ }^{17}$, Liang Qiao ${ }^{3}$, Xiuhua Jiang ${ }^{1}$, \\ Haiyu Wang', Guotao Zhong ${ }^{1}$, Beiling Li ${ }^{1}$, Jinjun Chen ${ }^{1,18 \wedge}$
}

\begin{abstract}
${ }^{1}$ Hepatology Unit, Department of Infectious Diseases, Nanfang Hospital, Southern Medical University, Guangzhou, China; ${ }^{2}$ Department of Hepatology, Chenzhou No. 1 People's Hospital, Chenzhou, China; ${ }^{3}$ Department of Gastroenterology, Ren Ji Hospital, School of Medicine, Shanghai Jiao Tong University, Shanghai, China; ${ }^{4}$ Department of Infectious Diseases, Southwest Hospital, Third Military Medical University (Army Medical University), Chongqing, China; ${ }^{5}$ Center of Integrative Medicine, Beijing Ditan Hospital, Capital Medical University, Beijing, China; ${ }^{6}$ Department of Infectious Diseases, Institute of Infection and Immunology, Union Hospital, Tongji Medical College, Huazhong University of Science and Technology, Wuhan, China; ${ }^{7}$ Department of Infectious Diseases, Hunan Key Laboratory of Viral Hepatitis, Xiangya Hospital, Central South University, Changsha, China; ${ }^{8}$ Department of Infectious Diseases, Hubei Clinical Research Center for Precise Diagnosis and Treatment of Liver Cancer, Taihe Hospital, Hubei University of Medicine, Hubei, China; ${ }^{9}$ Department of Hepatology, The First Hospital of Jilin University, Jilin, China; ${ }^{10}$ Department of Liver Intensive Care Unit, Shanghai Public Health Clinical Centre, Fudan University, Shanghai, China; ${ }^{11}$ Department of Infectious Diseases and Hepatology, The Second Hospital of Shandong University, Jinan, China; ${ }^{12}$ Infectious Disease Center, The First Affiliated Hospital of Xinjiang Medical University, Xinjiang, China; ${ }^{13}$ State Key Laboratory for Diagnosis and Treatment of Infectious Diseases, Collaborative Innovation Center for Diagnosis and Treatment of Infectious Disease, The First Affiliated Hospital, Zhejiang University School of Medicine, Hangzhou, China; ${ }^{14}$ Department of Infectious Diseases, Henan Provincial People's Hospital, Henan, China; ${ }^{15}$ Department of Infectious Diseases, The Third Affiliated Hospital, Sun Yat-sen University, Guangzhou, China; ${ }^{16}$ Department of Hepatology, Ningbo No. 2 Hospital, Ningbo, China; ${ }^{17}$ Clinical Research Center, Shanghai Jiao Tong University School of Medicine, Shanghai, China; ${ }^{18}$ Hepatology Unit, Zengcheng Branch, Nanfang Hospital, Southern Medical University, Guangzhou, China

Contributions: (I) Conception and design: B Li, J Chen, H Li, J Xia, X Wang, X Zheng, Y Huang, Z Meng, Y Gao, Z Qian, F Liu, X Lu, Y Shi, J Shang, J Liu, G Deng, Y Zheng, H Yan; (II) Administrative support: None; (III) Provision of study materials or patients: J Chen, H Li, J Xia, X Wang, X Zheng, Y Huang, Z Meng, Y Gao, Z Qian, F Liu, X Lu, Y Shi, J Shang, J Liu, G Deng; (IV) Collection and assembly of data: R Ouyang, B Li, X Jiang, H Wang, G Zhong, L Qiao; (V) Data analysis and interpretation: R Ouyang, W Zhang, L Qiao; (VI) Manuscript writing: All authors; (VII) Final approval of manuscript: All authors.

"These authors contributed equally to this work.

Correspondence to: Jinjun Chen. Hepatology Unit, Department of Infectious Diseases, Nanfang Hospital, Southern Medical University, No. 1838, Guangzhou Dadao Bei, Guangzhou 510515, China. Email: chjj@smu.edu.cn; Beiling Li. Hepatology Unit, Department of Infectious Diseases, Nanfang Hospital, Southern Medical University, No. 1838, Guangzhou Dadao Bei, Guangzhou 510515, China. Email: 1bling123@163.com.
\end{abstract}

Background Chronic liver diseases (CLD), including cirrhosis and non-cirrhotic liver diseases, are globally widespread and create a serious disease burden. Platelet count is a clinically accessible and affordable prognostic indicator of liver disease. We investigated the relationship between platelet count and 90-day prognosis in patients with acute-on-chronic liver diseases (AoCLD).

Methods: A total of 3,970 patients with AoCLD from the Chinese Acute-on-Chronic Liver Failure (CATCH-LIFE) study, which included two prospective multi-center cohorts, were included in the study. We grouped the patients according to the platelet count and analyzed the 90-day adverse outcome (death or liver transplantation).

Results: In the final analysis, 3,939 patients with AoCLD were included, of whom 2,802 had definite liver cirrhosis. The cumulative incidence of 90-day adverse outcomes in patients increased with the change of

\footnotetext{
$\wedge$ ORCID: 0000-0003-4275-9149.
} 
platelet group (log-rank $\mathrm{P}<0.001)$. From univariate and multivariate analyses, platelet count was inversely associated with the incidence of 90 -day adverse outcomes in patients ( $\mathrm{P}$ for trend $<0.001$ ). The group with platelet count $<20 \times 10^{9} / \mathrm{L}$ had the highest risk (odds ratio, 3.15; 95\% confidence interval, 1.59-6.25), with 21 $(36.8 \%)$ of these patients having adverse outcomes within 90 days. The risk of a 90 -day adverse outcome in patients increased by $5 \%$ for every $10 \times 10^{9} / \mathrm{L}$ decrease in platelet count below $210 \times 10^{9} / \mathrm{L}$.

Conclusions: Lower platelet count was associated with a higher incidence of 90-day adverse outcomes in patients with AoCLD. Even within the normal platelet count range, the risk of a 90-day adverse outcome in patients increased with decreases in platelet count.

Trial Registration: NCT02457637, NCT03641872.

Keywords: Platelet count; adverse outcome; chronic liver disease (CLD); prognosis; sequential organ failure assessment

Submitted Apr 25, 2021. Accepted for publication Jul 26, 2021.

doi: 10.21037/apm-21-1019

View this article at: https://dx.doi.org/10.21037/apm-21-1019

\section{Introduction}

Chronic liver diseases (CLDs) usually include cirrhosis and non-cirrhotic liver diseases, such as chronic viral hepatitis, alcoholic liver disease, and non-alcoholic fatty liver disease. As of 2017, about 1.5 billion people worldwide were affected by CLD, the digestive disease with the largest number of patients and which causes more than 1.3 million deaths a year $(1,2)$. In China alone, CLD poses a serious burden of disease, resulting in the deaths of about 154,000 patients a year $(3,4)$. Patients with CLD are often hospitalized because of acute hepatic injury or decompensation, which are termed acute-on-chronic liver diseases (AoCLD) (5). Early detection of disease changes and timely treatment are important measures to improve the prognosis of CLD.

Platelets, also called thrombocytes, are the smallest type of blood cell, and are produced by megakaryocytes in the bone marrow; they are active players in liver disease and inflammation (6,7). Among patients with CLD, those with cirrhosis usually experience thrombocytopenia due to multifactorial conditions. The degree of thrombocytopenia is proportional to the severity of liver disease $(8,9)$. Therefore, platelet count is often used in the diagnosis and evaluation of liver disease. For example, the sequential organ failure assessment (SOFA) score and the chronic liver failure-sequential organ failure assessment (CLIF-SOFA) score, used for the assessment of acute-on-chronic liver failure (ACLF), the fibrosis 4 score (FIB-4) and aspartate aminotransferase to platelet ratio index (APRI), used for liver fibrosis evaluation, and the Baveno VI criteria for portal hypertension all use platelet count as an indicator
(10-14). Recently, platelet count has also been used in a new score to predict hepatocellular carcinoma development in patients with chronic hepatitis (15). However, few clinical studies on platelet-related prognosis have been performed in the huge population with AoCLD, who are patients with CLD requiring active medical intervention. In this study, we aimed to analyze the relationship between platelet counts and 90-day adverse outcomes in patients with AoCLD and to evaluate prognosis based on platelet count. We present the following article in accordance with the STROBE reporting checklist (available at https://dx.doi. org/10.21037/apm-21-1019).

\section{Methods}

\section{Patients}

Patients were from the Chinese Acute-on-Chronic Liver Failure (CATCH-LIFE) study, which included two prospective multi-center cohorts with AoCLD. There were 2,600 patients in the investigation cohort (enrollment initiated in January 2015 and ended in December 2016) and 1,370 patients in the validation cohort (enrollment initiated in July 2018 and ended in January 2019), recruited from 15 tertiary hospitals in China $(5,16,17)$. The study was conducted in accordance with the Declaration of Helsinki (as revised in 2013) and was approved by the Renji Hospital Ethics Committee of Shanghai Jiaotong University School of Medicine (No. 2014-148K and 2016-142K). The study was registered at www.clinicaltrials.gov (NCT02457637, NCT03641872). All patients gave their informed consent 


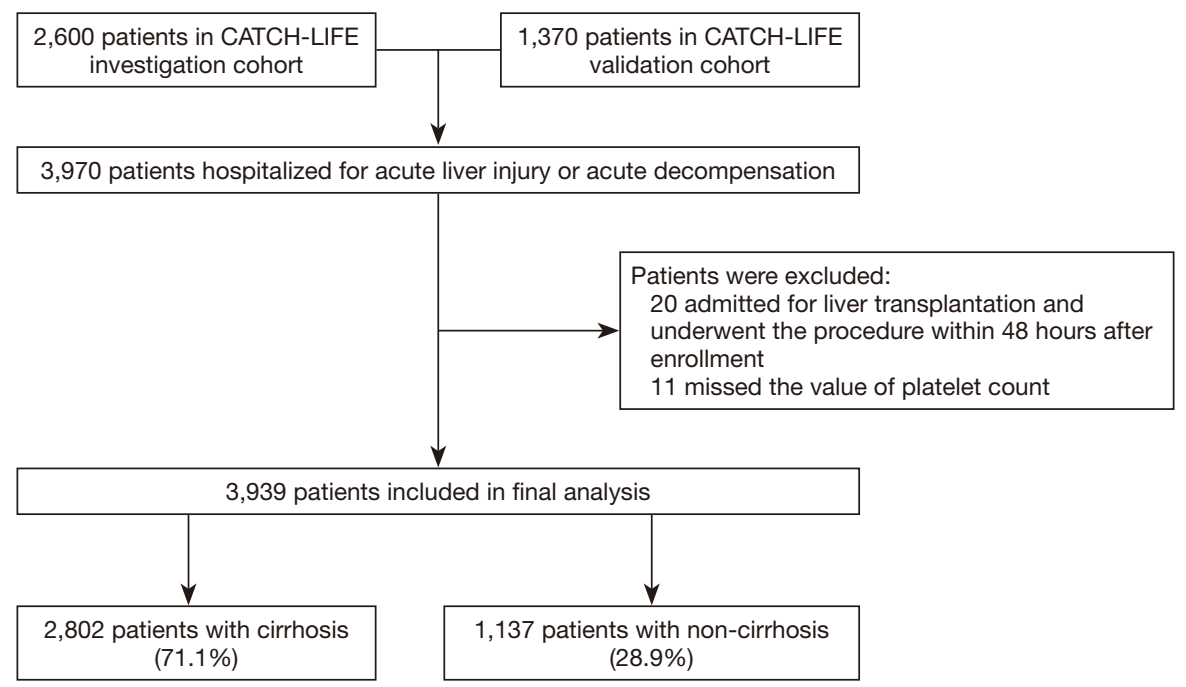

Figure 1 Screening and enrolment of patients. CATCH-LIFE, Chinese Acute-on-Chronic Liver Failure.

prior to their inclusion in the study.

In this study, CLD was defined as cirrhosis or noncirrhotic liver disease with a history of liver dysfunction lasting more than 6 months. Cirrhosis was diagnosed based on computed tomography or magnetic resonance imaging, laboratory tests, clinical symptoms, and history of liver disease. Acute exacerbation was defined as acute hepatic injury [alanine aminotransferase (ALT) or aspartate aminotransferase (AST) $>3 \times$ upper limit of normal or total bilirubin $>2 \times$ upper limit of normal] within 1 week before enrollment or acute decompensation [ascites, hepatic encephalopathy (HE), bacterial infection, or gastrointestinal bleeding] within 1 month before enrollment $(5,16,17)$.

We included 3,970 patients in this analysis. Twenty patients who were admitted for transplantation and underwent the procedure within 48 hours after enrollment, and 11 patients with missing platelet count data were excluded. The final analysis included 3,939 patients (Figure 1).

\section{Data collection}

We collected the following demographic and clinical information on admission: age; sex; etiologies of liver disease and acute decompensation events; laboratory parameters; scores [Child-Turcotte-Pugh (CTP); CLIFSOFA; Model for End-Stage Liver Disease (MELD), and MELD-sodium (MELD-Na)]; and prognosis (allcause mortality was considered the endpoint; liver transplantation and loss to follow-up were considered censoring events). Diagnosis of acute-on-chronic liver failure (ACLF) was performed according to the European Association for the Study of Liver-Chronic Liver Failure (EASL-CLIF) criteria (11).

\section{Outcomes}

The outcome was an adverse outcome [death or liver transplantation (LT)] within 90 days.

\section{Statistical analysis}

Continuous variables were analyzed using Student's $t$-test, Mann-Whitney's U test, or Kruskal-Wallis test among different groups and expressed as medians with interquartile range (IQR). Categorical variables were compared with the chi-squared or Fisher's exact tests and expressed as number and percentage. The effect of prognostic variables on 90-day adverse outcome was analyzed using the KaplanMeier method and compared by log-rank test.

Patients were divided into five groups (platelet count $<20 \times 10^{9} / \mathrm{L}, 20-50 \times 10^{9} / \mathrm{L}, 50-100 \times 10^{9} / \mathrm{L}, 100-150 \times 10^{9} / \mathrm{L}$, and $\geq 150 \times 10^{9} / \mathrm{L}$ ) based on SOFA score and the lower limit of normal platelet count. Multivariate logistic regression by a backward stepwise method was used to assess the odds ratio (OR) and 95\% confidence interval (CI) for 90-day adverse outcome and platelet count (as a continuous variable and a categorical variable). In addition, logistic regression was used to assess the statistical significance of trends based 
on variables containing the median value for each group (18). We adjusted for several factors in our logistic models. In model 1, we adjusted for age, sex, cirrhosis, and etiology. In model 2, we adjusted for model 1 plus various laboratory parameters (ALT, white blood cell count, hemoglobin, sodium, total bilirubin, international normalized ratio, and creatinine). In model 3, we adjusted for model 2 plus acute decompensation events, such as gastrointestinal bleeding, infection, ascites, and HE. Sensitivity analysis included patients after multiple imputations of missing values (Figure S1).

A generalized additive model and smooth curve fitting were performed to characterize the shape of the relationship between platelet count and the incidence of 90-day adverse outcome. A two-sided significance level of $<0.05$ was used to evaluate statistical significance. Statistical analyses were performed using SPSS (version 25.0; SPSS Inc, Chicago, IL, USA) or R (version 4.0.2; http://www.r-project.org, Vienna, Austria).

\section{Results}

\section{Patients and baseline characteristics}

Of 3,939 patients included in the final analysis, 2,802 had cirrhosis, and the remaining 1,137 patients did not have definite liver cirrhosis (Figure 1).

Figure S2 shows the frequency of platelet counts. Baseline characteristics for the platelet groups are presented in Table 1. There were significant differences $(\mathrm{P}<0.05)$ in demographic data, acute decompensation events, laboratory data, and scores among the groups.

\section{0-day outcomes}

No patients were lost to follow-up within 90 days. The number of outcomes per group is displayed in Table 1 . Patients with 90-day adverse outcomes had lower median platelet count than those without (median $76 \times 10^{9} / \mathrm{L} v s$. $\left.99 \times 10^{9} / \mathrm{L}, \mathrm{P}<0.001\right)$. The median platelet count in the acute decompensation subgroup was lower in the present group than in the absent group $(\mathrm{P}<0.001)$ (Figure S3). Patients in group 1 (platelet count $<20 \times 10^{9} / \mathrm{L}$ ) had the worst prognosis, with $21(36.8 \%)$ patients having 90-day adverse outcomes. The incidence of adverse outcomes was lowest in group 5 (platelet count $\geq 150 \times 10^{9} / \mathrm{L}$ ), with $66(7.1 \%)$ patients who died and 31 (3.3\%) who had liver transplantation. The LTfree mortality was $20.0 \%, 17.8 \%, 16.0 \%, 13.0 \%$, and $7.3 \%$ in groups 1, 2, 3, 4, and 5, respectively.

On Kaplan-Meier analysis, the cumulative incidence of 90-day adverse outcomes in patients with AoCLD increased with the change of platelet group (log-rank $\mathrm{P}<0.001)$ (Figure 2). There were also differences between each group in patients with cirrhosis or non-cirrhosis. The difference was significant in cirrhosis patients without ACLF, but not significant in patients with ACLF (Figure S4).

\section{Univariate and multivariate analysis for 90-day adverse outcomes}

Univariate and multivariate analysis for 90-day adverse outcomes is presented in Table 2. We constructed an unadjusted model and three adjusted models to evaluate the relationship between platelet count and 90-day adverse outcomes. The results of univariate and multivariate analysis were similar: platelet count was inversely associated with the incidence of 90-day adverse outcomes (P for trend $<0.001$ ). Compared with group 5 in model 3 (adjusted for the most variables), the risk in each group gradually increased, and group 1 had the highest risk (OR, 3.15; 95\% CI, 1.59-6.25). As a continuous variable (per $10 \times 10^{9} / \mathrm{L}$ decrease), platelet count was also found to be independently associated with 90-day adverse outcomes (OR, 1.02; 95\% CI, 1.00-1.04). In the subgroup analysis of cirrhosis and non-cirrhosis, we found the same trend results as in the overall analysis. Furthermore, the same trend was seen in the non-ACLF subgroup in cirrhosis (Table S1). Sensitivity analysis after multiple imputations of missing values obtained results consistent with the above analysis (Table S2).

\section{Associations between platelet count and the incidence of 90-day adverse outcomes}

A generalized additive model with $\mathrm{P}$-spline smoothers was performed to characterize the shape of the relationship between platelet count and the incidence of 90-day adverse outcomes in adjusted model 3 (Figure S5). The adjusted effect of platelet count between 0 and $210 \times 10^{9} / \mathrm{L}$ on $\log$ odds of 90 -day adverse outcomes was nearly linear (Figure 3), with an adjusted OR of 1.05 (95\% CI, $1.03-1.08 ; \mathrm{P}<0.001)($ Table 3$)$ associated with a $10 \times 10^{9} / \mathrm{L}$ decrease in platelet count at any level between 0 and $210 \times 10^{9} / \mathrm{L}$ (e.g., a patient with platelet count of $140 \times 10^{9} / \mathrm{L}$ had a $5 \%$ higher adjusted odds of adverse outcome than one with platelet count of $150 \times 10^{9} / \mathrm{L}$, and so on throughout the entire range) (19). 
Table 1 Patient characteristics for each platelet group

\begin{tabular}{|c|c|c|c|c|c|c|}
\hline Variables $^{\mathrm{a}}, 10^{9} / \mathrm{L}$ & PLT $<20, N=57$ & $20 \leq \mathrm{PLT}<50, \mathrm{~N}=660$ & $\begin{array}{c}50 \leq \mathrm{PLT}<100 \\
\mathrm{~N}=1,390\end{array}$ & $\begin{array}{c}100 \leq \mathrm{PLT}<150 \\
\mathrm{~N}=897\end{array}$ & $\mathrm{PLT} \geq 150, \mathrm{~N}=935$ & $P$ value \\
\hline Age, years & $49.0(41.0-58.0)$ & $51.0(44.3-59.0)$ & $50.0(42.0-59.0)$ & $47.0(37.0-57.0)$ & $43.0(34.0-53.0)$ & $<0.001$ \\
\hline Etiology, n (\%) & & & & & & $<0.001$ \\
\hline HBV-alcoholic & $3(5.3)$ & $50(7.6)$ & $139(10.0)$ & $85(9.5)$ & $76(8.1)$ & \\
\hline $\mathrm{HCV}$ & $2(3.5)$ & $27(4.1)$ & $64(4.6)$ & $20(2.2)$ & $19(2.0)$ & \\
\hline Others & $16(28.1)$ & $117(17.7)$ & $262(18.8)$ & 205 (22.9) & $276(29.5)$ & \\
\hline Non-HE & $50(87.7)$ & $579(87.7)$ & $1,257(90.4)$ & $834(93.0)$ & $880(94.1)$ & \\
\hline Grade 1-2 & $5(8.8)$ & $65(9.8)$ & $104(7.5)$ & $50(5.6)$ & $40(4.3)$ & \\
\hline Grade 3-4 & $2(3.5)$ & $16(2.4)$ & $29(2.1)$ & $13(1.4)$ & $15(1.6)$ & \\
\hline Infection & $13(22.8)$ & $164(24.8)$ & $342(24.6)$ & $169(18.8)$ & $153(16.4)$ & $<0.001$ \\
\hline Ascites & $37(64.9)$ & $430(65.2)$ & $770(55.4)$ & 358 (39.9) & $252(27.0)$ & $<0.001$ \\
\hline $\begin{array}{l}\text { Gastrointestinal } \\
\text { bleeding }\end{array}$ & $14(24.6)$ & $156(23.6)$ & $256(18.4)$ & $84(9.4)$ & $68(7.3)$ & $<0.001$ \\
\hline ALT, IU/L & $36.1(19.0-77.2)$ & $36.2(22.0-74.6)$ & $64.9(29.1-214.0)$ & $226.0(58.0-662.6)$ & 259.5 (67.1-714.0) & $<0.001$ \\
\hline AST, IU/L & $56.0(30.5-110.5)$ & $51.5(32.0-98.6)$ & $89.5(44.8-210.3)$ & $175.0(76.0-430.1)$ & $171.0(72.0-425.1)$ & $<0.001$ \\
\hline WBC, $10^{9} / \mathrm{L}$ & $2.7(2.0-4.7)$ & $3.3(2.3-4.9)$ & $4.5(3.4-6.4)$ & $5.5(4.3-7.4)$ & $6.2(4.9-8.4)$ & $<0.001$ \\
\hline Hemoglobin, g/L & $91.0(73.5-111.0)$ & $103.0(82.0-118.0)$ & $114.0(92.0-129.0)$ & $127.0(106.5-143.0)$ & $131.0(111.0-146.0)$ & $<0.001$ \\
\hline Sodium, mmol/L & $136.5(132.0-139.2)$ & $138.0(134.6-140.6)$ & $138.0(134.9-140.8)$ & $138.4(136.0-140.7)$ & $139.0(136.2-141.0)$ & $<0.001$ \\
\hline CTP score & $10.0(8.0-11.5)$ & $10.0(8.0-11.0)$ & $9.0(7.8-11.0)$ & $9.0(7.0-10.0)$ & $7.0(6.0-9.0)$ & $<0.001$ \\
\hline MELD score & $16.0(12.0-24.0)$ & $16.0(11.0-21.8)$ & $16.0(11.0-23.0)$ & $16.0(10.0-23.0)$ & $12.0(8.0-19.0)$ & $<0.001$ \\
\hline MELD-Na score & $19.0(14.5-25.5)$ & $17.0(13.0-24.0)$ & $18.0(12.0-25.0)$ & $18.0(12.0-25.0)$ & $14.0(9.0-22.0)$ & $<0.001$ \\
\hline CLIF-SOFA score & $7.0(6.0-8.0)$ & $5.0(4.0-7.0)$ & $5.0(3.0-7.0)$ & $5.0(3.0-7.0)$ & $4.0(2.0-6.0)$ & $<0.001$ \\
\hline EASL-ACLF & $10(17.5)$ & $96(14.5)$ & 166 (11.9) & $90(10.0)$ & $54(5.8)$ & $<0.001$ \\
\hline
\end{tabular}

Table 1 (continued) 
Table 1 (continued)

\begin{tabular}{|c|c|c|c|c|c|c|}
\hline Variables $^{\mathrm{a}}, 10^{9} / \mathrm{L}$ & PLT $<20, N=57$ & $20 \leq \mathrm{PLT}<50, \mathrm{~N}=660$ & $\begin{array}{c}50 \leq \mathrm{PLT}<100 \\
\mathrm{~N}=1,390\end{array}$ & $\begin{array}{c}100 \leq \mathrm{PLT}<150 \\
\mathrm{~N}=897\end{array}$ & $\mathrm{PLT} \geq 150, \mathrm{~N}=935$ & $P$ value ${ }^{b}$ \\
\hline \multicolumn{7}{|l|}{ Outcome, n (\%) } \\
\hline $\begin{array}{l}\text { 90-day adverse } \\
\text { outcome }\end{array}$ & $21(36.8)$ & $166(25.2)$ & $298(21.4)$ & $246(16.3)$ & $97(10.4)$ & $<0.001$ \\
\hline Death & $9(15.8)$ & $107(16.2)$ & $208(15.0)$ & $112(12.5)$ & $66(7.1)$ & \\
\hline LT & $12(21.1)$ & $59(8.9)$ & $90(6.5)$ & $34(3.8)$ & $31(3.3)$ & \\
\hline
\end{tabular}

${ }^{a}$, continuous data are presented as median (25th-75th percentiles); ${ }^{b}$, comparison between patients in the five groups. PLT, platelet count; HBV, hepatitis B virus; HCV, hepatitis C virus; HE, hepatic encephalopathy; INR, international normalized ratio; BUN, blood urea nitrogen; ALT, aspartate aminotransferase; AST, alanine aminotransferase; WBC, white blood cell; CTP, child-turcotte-pugh; MELD, model for end-stage liver disease; MELD-Na, model for end-stage liver disease-sodium; CLIF-SOFA, chronic liver failure-sequential organ failure assessment; EASL, European Association for the Study of Liver; ACLF, acute-on-chronic liver failure; LT, liver transplantation.

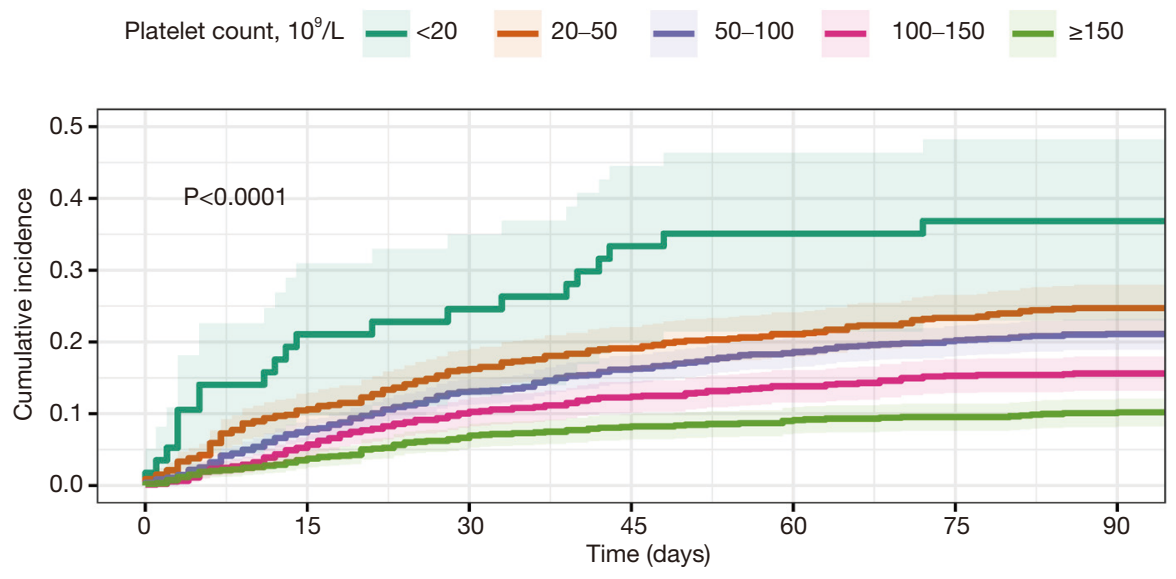

\begin{tabular}{|c|c|c|c|c|c|c|c|}
\hline \multicolumn{8}{|c|}{ Number at risk } \\
\hline$<20$ & 57 & 45 & 43 & 38 & 37 & 36 & 36 \\
\hline $20-50$ & 660 & 590 & 553 & 533 & 520 & 505 & 492 \\
\hline $50-100$ & 1390 & 1287 & 1208 & 1163 & 1131 & 1107 & 1090 \\
\hline $100-150$ & 897 & 850 & 806 & 786 & 772 & 759 & 754 \\
\hline$\geq 150$ & 935 & 902 & 871 & 855 & 845 & 840 & 834 \\
\hline
\end{tabular}

Figure 2 Kaplan-Meier graph of 90-day adverse outcome stratified by platelet groups.

We dichotomized patients in the study into "low" vs. "not low" platelet groups using various cut point increments of $10 \times 10^{9} / \mathrm{L}$ starting from $200 \times 10^{9} / \mathrm{L}$ (Figure 4 ). Logistic regression adjusted model 3 was used to estimate the OR of 90-day adverse outcomes between the two groups. We found that as platelet count rose to $200 \times 10^{9} / \mathrm{L}$, the "low" group always had a higher risk of 90-day adverse outcomes than the "not low" group $(\mathrm{P}<0.05)$. Patients with platelet count below $20 \times 10^{9} / \mathrm{L}$ had the highest adjusted odds of adverse outcomes (OR, 2.10; 95\% CI, 1.12-3.95).

\section{Discussion}

The patients with AoCLD in this study included not only patients with ACLF but also those who were hospitalized due to acute hepatic injury or decompensation and did 
Table 2 Univariate and multivariate logistic regression analysis of 90-day adverse outcome

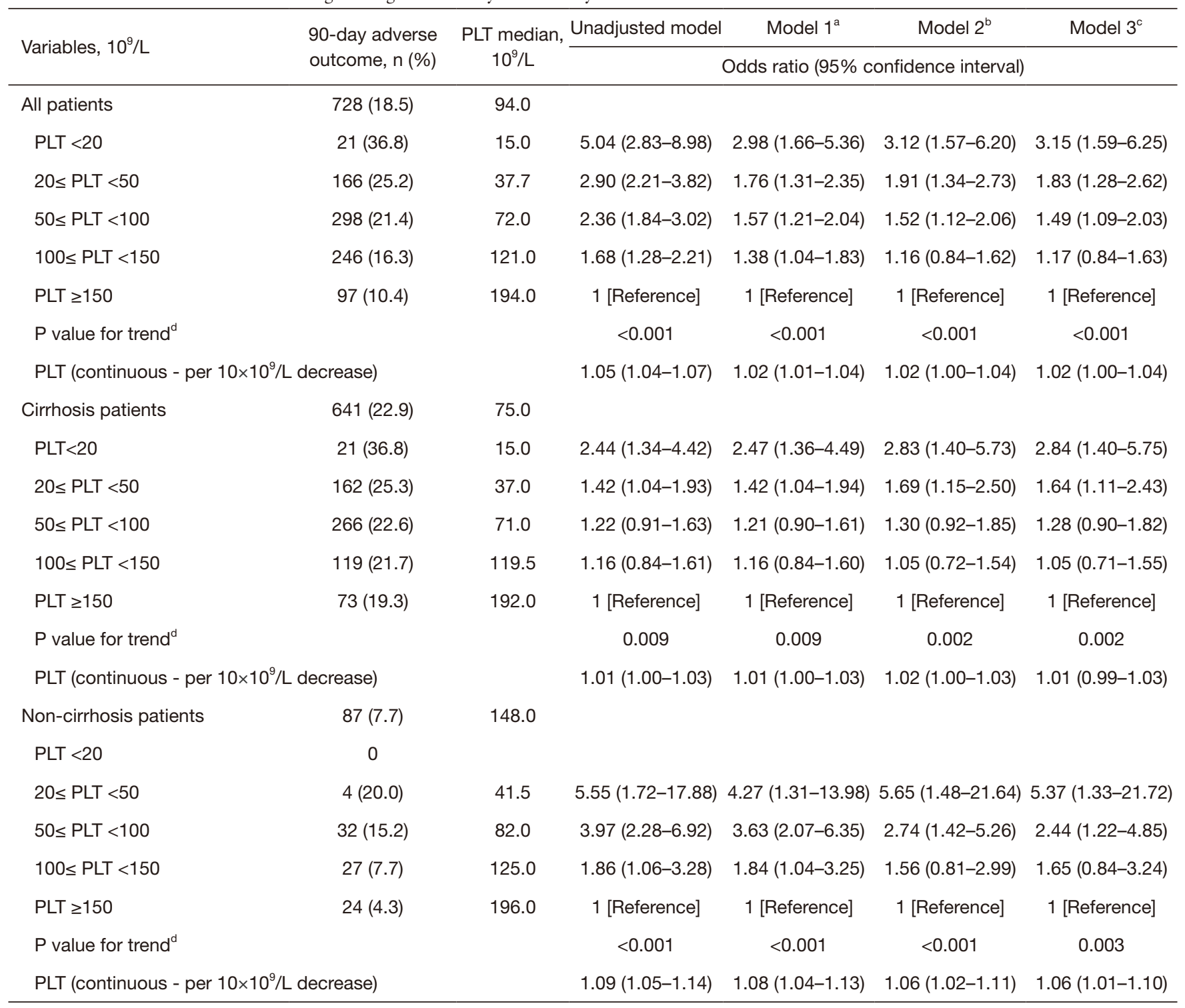

${ }^{a}$, model 1: adjusted for age, sex, cirrhosis, and etiology; ${ }^{b}$, model 2: adjusted for model 1 plus laboratory variables (ALT, WBC, hemoglobin, sodium, total bilirubin, INR, and creatinine); ' , model 3: adjusted for model 2 plus AD (gastrointestinal bleeding, infection, ascites, and HE); ${ }^{\text {, }}$ test for trend based on variable containing median value for each group. PLT, platelet count; ALT, aspartate aminotransferase; WBC, white blood cell; INR, international normalized ratio; $A D$, acute decompensation; HE, hepatic encephalopathy.

not meet the ACLF standard, which is more in line with the overall situation of patients who need treatment in clinical practice. This study is the first to use multicenter prospective cohort data to clarify the relationship between platelet count and the 90-day prognosis of patients with AoCLD. Lower platelet count was associated with 90-day adverse outcomes of patients with AoCLD, among whom patients with platelet count below normal had worse outcomes than those whose platelet count was above normal, with a further decrease resulting in a worse outcome. From previous studies, we knew that the degree of platelet count reduction is related to the severity of liver disease, and when it is below a certain threshold, the risk of bleeding increases significantly $(9,20)$. Moreover, low platelet count could adversely affect the treatment of CLD, limiting the ability to perform therapy and delaying planned 


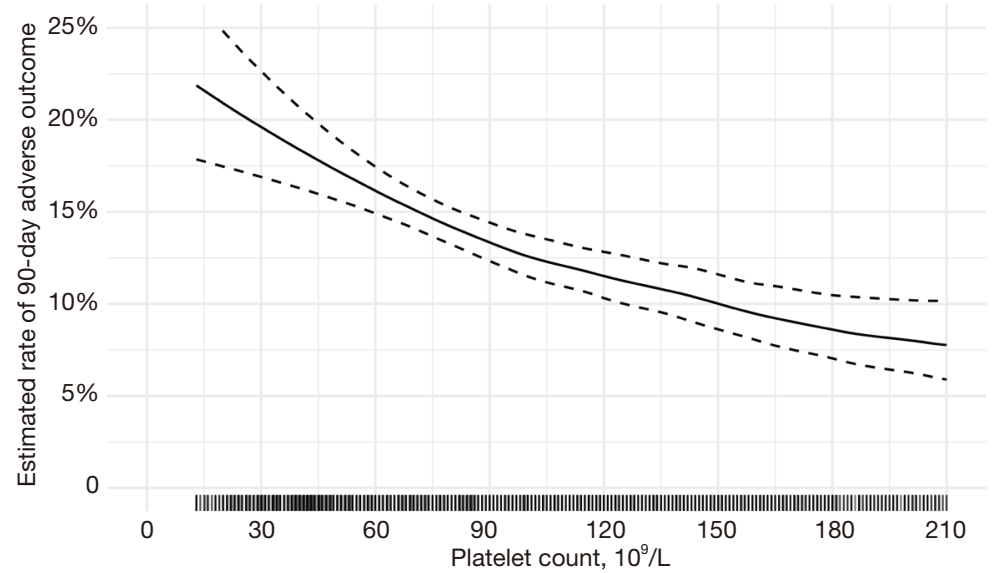

Figure 3 Association of platelet count and the incidence of 90-day adverse outcome in adjusted model 3: age, sex, cirrhosis, etiology, laboratory variables (ALT, WBC, hemoglobin, sodium, total bilirubin, INR, creatinine), and AD (gastrointestinal bleeding, infection, ascites, HE). ALT, aspartate aminotransferase; WBC, white blood cell; INR, international normalized ratio; AD, acute decompensation; HE, hepatic encephalopathy.

Table 3 Multivariate logistic regression analysis of 90 -day adverse outcome (adjusts for model 3, platelet count $<210 \times 10^{9} / \mathrm{L}$ )

\begin{tabular}{lcc}
\hline PLT (continuous) & $\begin{array}{c}\text { Odds ratio }(95 \% \\
\text { confidence interval) }\end{array}$ & P value \\
\hline All patients & $1.05(1.03-1.08)$ & $<0.001$ \\
Per $10 \times 10^{9} / \mathrm{L}$ decrease & $1.11(1.05-1.17)$ & $<0.001$ \\
Per $20 \times 10^{9} / \mathrm{L}$ decrease & & \\
Cirrhosis patients & $1.05(1.02-1.08)$ & $<0.001$ \\
Per $10 \times 10^{\circ} / \mathrm{L}$ decrease & $1.10(1.04-1.16)$ & $<0.001$ \\
Per $20 \times 10^{9} / \mathrm{L}$ decrease & & \\
Non-cirrhosis patients & $1.08(1.01-1.16)$ & 0.027 \\
Per $10 \times 10^{\circ} / \mathrm{L}$ decrease & $1.17(1.02-1.35)$ & 0.027 \\
\hline Per $20 \times 10^{\circ} / \mathrm{L}$ decrease &
\end{tabular}

Model 1: adjusted for age, sex, cirrhosis, and etiology; Model 2: adjusted for model 1 plus laboratory variables (ALT, WBC, hemoglobin, sodium, total bilirubin, INR, and creatinine); Model 3: adjusted for model 2 plus AD (gastrointestinal bleeding, infection, ascites, and HE). PLT, platelet count; ALT, aspartate aminotransferase; WBC, white blood cell; INR, international normalized ratio; $A D$, acute decompensation; $H E$, hepatic encephalopathy.

surgical/diagnostic procedures due to increased bleeding risk (21).

In the study, we used the platelet scoring standard from the evaluation of coagulation failure in the SOFA score for grouping. The SOFA score is widely used to describe organ dysfunction/failure in general intensive care units, and it is also used for the prognosis of patients with severe liver disease $(11,22)$. The results confirmed that platelet grading in the SOFA score has clinical significance for the prognosis of patients with CLD. Moreover, the numerical values of the four score thresholds are relatively easy to remember and convenient for clinical use.

The lower limit of the normal range of platelet count is generally $150 \times 10^{9} / \mathrm{L}$, and below this value is defined as thrombocytopenia. The prevalence of thrombocytopenia has been observed in up to $76 \%$ of patients with CLD, which is almost consistent with our results (21). However, it should be noted that the current normal range of platelet count is not necessarily applicable in patients with CLD. In our study, we found that platelet count was nearly negatively linearly correlated with adverse outcomes from below $210 \times 10^{9} / \mathrm{L}$, which is still within the normal range. Therefore, any two patients with a platelet count difference of $10 \times 10^{9} / \mathrm{L}$, in the range below $210 \times 10^{9} / \mathrm{L}$, differed in the adjusted 90 -day adverse outcome rate by $5 \%$. Our results also showed that as platelet count cutoff increased to $200 \times 10^{9} / \mathrm{L}$, the "low" group always had a higher risk of 90-day adverse outcomes than the "not-low" group. In Figure S5, we found that when the platelet count was $>210 \times 10^{9} / \mathrm{L}$, the incidence of 90 -day adverse outcomes increases, but the increase was within limit, and the CI represented by the dotted line was 


$\begin{array}{cc}\text { Platelet count, } 10^{9} / \mathrm{L} & \text { Odds ratio }(95 \% \text { Cl) } \\ <20 \text { vs. } \geq 20 & 2.10(1.12-3.95) \\ <30 \text { vs. } \geq 30 & 1.66(1.16-2.47) \\ <40 \text { vs. } \geq 40 & 1.52(1.15-2.01) \\ <50 \text { vs. } \geq 50 & 1.44(1.13-1.83) \\ <60 \text { vs. } \geq 60 & 1.40(1.12-1.75) \\ <70 \text { vs. } \geq 70 & 1.37(1.11-1.70) \\ <80 \text { vs. } \geq 80 & 1.32(1.07-1.63) \\ <90 \text { vs. } \geq 90 & 1.32(1.06-1.63) \\ <100 \text { vs. } \geq 100 & 1.46(1.17-1.82) \\ <110 \text { vs. } \geq 110 & 1.48(1.17-1.86) \\ <120 \text { vs. } \geq 120 & 1.48(1.17-1.88) \\ <130 \text { vs. } \geq 130 & 1.46(1.13-1.87) \\ <140 \text { vs. } \geq 140 & 1.50(1.15-1.96) \\ <150 \text { vs. } \geq 150 & 1.42(1.07-1.89) \\ <160 \text { vs. } \geq 160 & 1.36(1.00-1.83) \\ <170 \text { vs. } \geq 170 & 1.49(1.07-2.08) \\ <180 \text { vs. } \geq 180 & 1.58(1.10-2.28) \\ <190 \text { vs. } \geq 190 & 1.49(1.01-2.18) \\ <200 \text { vs. } \geq 200 & 1.55(1.02-2.35)\end{array}$

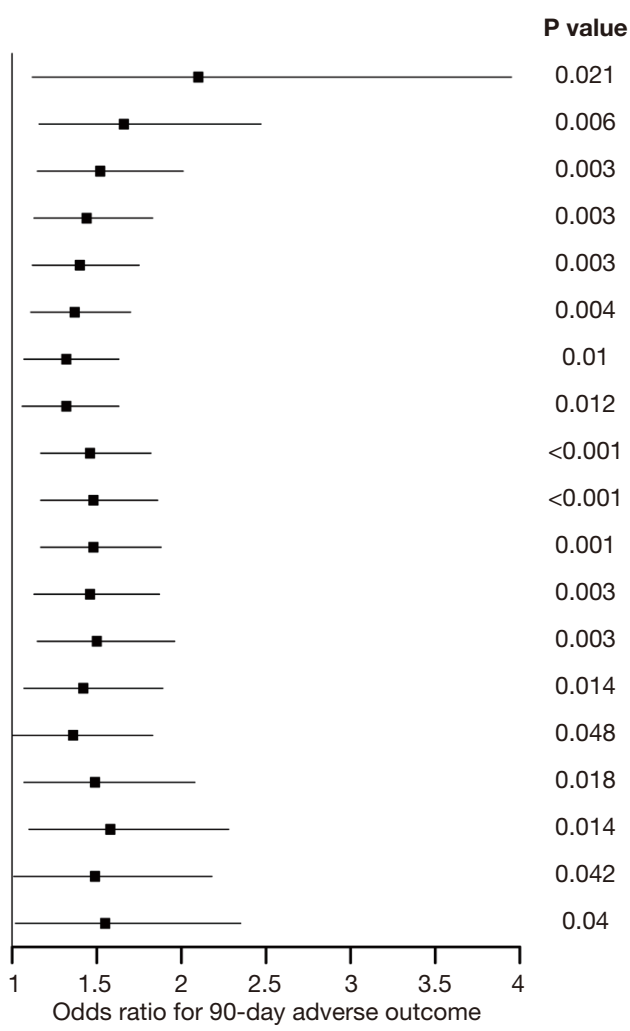

Figure 4 Platelet count and adjusted odds ratio of 90-day adverse outcomes. Adjusted for age, sex, cirrhosis, etiology, laboratory variables (ALT, WBC, hemoglobin, sodium, total bilirubin, INR, creatinine), and AD (gastrointestinal bleeding, infection, ascites, HE). CI, confidence interval; ALT, aspartate aminotransferase; WBC, white blood cell; INR, international normalized ratio; AD, acute decompensation; HE hepatic encephalopathy.

also significantly larger. In addition, only 355 patients had platelet counts $>210 \times 10^{9} / \mathrm{L}$ in this study, which was $<10 \%$ of the total. Among them, 31 patients with adverse outcomes accounted for less than $5 \%$ of the overall adverse outcomes, with a relatively limited impact on the overall results. Therefore, the decrease in platelet counts in the overall trend was associated with an increase in the incidence of adverse outcomes. In AoCLD, we must be aware that the prognosis of patients with different platelet counts is different even when platelet counts are within the normal range.

In our study, all patients with platelet count $<20 \times 10^{9} / \mathrm{L}$ had cirrhosis, and they had the highest incidence of 90-day adverse outcomes whether with ACLF or not. Patients often required platelet transfusion in clinical practice and were not suitable for invasive surgery (21). In the CLIF-SOFA score that evolved from the SOFA score, we also found that platelet count $<20 \times 10^{9} / \mathrm{L}$ was associated with the highest score in coagulation failure (11). These results remind us to pay careful attention to such patients in clinical practice. From another perspective, patients without ACLF on admission might have pre-ACLF, in which two major pathophysiological mechanisms (systemic inflammation and portal hypertension) led to adverse outcomes (23). This situation would be in accordance with the patients in our study being admitted to the hospital due to acute hepatic injury or decompensation and with the 90-day prognosis assessment. Although such patients were only a few, further research might find some new knowledge about pre-ACLF.

Many non-invasive scores of liver fibrosis use the platelet count as an indicator because it is an active player in liver inflammation and fibrosis, which are related to the prognosis of CLD $(7,12,13)$. In our subgroup analysis of patients with non-cirrhosis, we found that platelet count was associated with 90-day adverse outcomes, which further proved its applicability in the evaluation of the prognosis of patients with non-cirrhosis. 
Because platelet count is associated with hypersplenism, it is also widely used in the diagnosis of portal hypertension. The Baveno VI Consensus had recommended that patients with platelet count $>150 \times 10^{9} / \mathrm{L}$ and liver stiffness measurement $<20 \mathrm{kPa}$ can relatively safely avoid screening endoscopy because only $5 \%$ of high-risk varices are missed (14). A recent study found that platelet count $>105 \times 10^{9} / \mathrm{L}$ could be used as an indicator to safely avoid more screening endoscopies in patients with hepatitis $\mathrm{B}$ virus-related compensated cirrhosis on antiviral therapy. It showed the potential of platelet count alone to identify patients at risk of portal hypertension (24), which was in line with our finding that patients with acute decompensation events, including gastrointestinal bleeding and ascites, have lower median platelet count.

Severe thrombocytopenia is often associated with severe complications of CLD, and it might be the latter, rather than thrombocytopenia itself, that ultimately determines the prognosis (25). Thus, the main reason for using plateletincreasing drugs in some studies was to reduce the need for platelet transfusions and the risk of bleeding during invasive surgery, rather than improve the prognosis of the disease in patients with CLD $(9,20,26,27)$. In addition, there was insufficient evidence to prove that thrombocytopenia can be treated by either platelet transfusion, splenic embolism, splenectomy, or placement of a Transjugular intrahepatic portosystemic stent shunt (TIPSS) to improve the longterm prognosis of CLD; these treatment measures also had several limitations and disadvantages (28).

In general, platelet count is a good indicator to help distinguish the natural course of CLD and assess the prognosis. Platelet count is a routine blood test, clinically accessible and affordable, even in underdeveloped areas; therefore, its use in assessing the condition of AoCLD is easy to adopt.

\section{Limitations}

Our study had several limitations. First, this study was designed to be observational, and the correlation between the change of platelet count and the prognosis after specific treatment could not be obtained. Second, the study used baseline data, but patients' previous treatment (such as platelet transfusion) was likely to impact platelet count at admission. Furthermore, the relationship between the dynamic changes of platelet count and the prognosis of patients with AoCLD was not analyzed, although some studies have found such a relationship $(29,30)$.

\section{Conclusions}

Lower platelet count was associated with the 90-day adverse outcome of patients with AoCLD, among whom patients with platelet count below normal had worse outcomes than those with platelet count above normal. Even within the normal range, the risk of a 90-day adverse outcome in patients increased by $5 \%$ for each $10 \times 10^{9} / \mathrm{L}$ decrease in platelet count below $210 \times 10^{9} / \mathrm{L}$.

\section{Acknowledgments}

We thank the following Chinese (Acute on) Chronic Liver Failure Consortium (Ch-CLIF.C) members and participants for the contributions to this study: Department of Gastroenterology, Ren Ji Hospital, School of Medicine, Shanghai Jiao Tong University-Shan Yin, Wenyi Gu, Yan Zhang, Tongyu Wang, Dandan Wu, Fuchen Dong, Bo Zeng, Liuying Chen, Shijin Wang; Centre of Integrative Medicine, Beijing Ditan Hospital, Capital Medical University-Qun Zhang, Yixin Hou, Yuxin Li, Yunyi Huang; Department of Infectious Diseases, Southwest Hospital, Third Military Medical University (Army Medical University)_Shuning Sun, Wenting Tan, Xiaomei Xiang, Yunjie Dan; Department of Infectious Disease, Hunan Key Laboratory of Viral Hepatitis, Xiangya Hospital, Central South University_-Jun Chen, Chengjin Liao, Xiaoxiao Liu; Department of Infectious Diseases, Institute of Infection and Immunology, Union Hospital, Tongji Medical College, Huazhong University of Science and Technology_Jing Liu, Ling Xu, Shue Xiong, Yan Xiong, Congcong Zou; Hepatology Unit, Department of Infectious Diseases, Nanfang Hospital, Southern Medical University_Congyan Zhu; Department of Hepatology, First Hospital of Jilin University—Chang Jiang, Xiaoyu Wen, Na Gao, Chunyan Liu; Department of Infectious Disease, Taihe Hospital, Hubei University of Medicine-Qing Lei, Sen Luo; Department of Infectious Disease, The First Hospital of Zhejiang University-Haotang Ren; Department of Liver Intensive Care Unit, Shanghai Public Health Clinical Centre, Fudan University-Xue Mei, Jiefei Wang, Liujuan Ji; Department of Infectious Diseases and Hepatology, Second Hospital of Shandong University-Tao Li, Xuanqiong Fang, Jing Li, Ziyu Wang; Liver Disease Centre, First Affiliated Hospital of Xinjiang Medical UniversityRongjiong Zheng, Fangrong Jie, Nan Li; Department of Infectious Disease, Henan Provincial People's HospitalHuiming Jin; Infectious Disease Center, Affiliated Hospital 
of Logistics University of People's Armed Police ForceHai Li, Qing Zhang, Xuequn Zheng; and Department of Infectious Disease, Fuzhou General Hospital of Nanjing Military Command-Shaoyang Wang, Taofa Lin.

Funding: This research was supported by the National Science and Technology Major Project (No. 2018ZX10723203, 2018ZX10302206), National Natural Science Foundation of China (No. 82070650, 81930061), National Key Research and Development Program of China (No. 2017YFC0908100), Local Innovative and Research Teams Project of Guangdong Pearl River Talents Program (No. 2017BT01S131), Clinical Research Program of Nanfang Hospital, Southern Medical University (No. 2018CR037, 2020CR022, 2020CR026), Clinical Research Startup Program of Southern Medical University by Highlevel University Construction Funding of Guangdong Provincial Department of Education (No. LC2019ZD006) and President Foundation of Nanfang Hospital, Southern Medical University (No. 2019Z003).

\section{Footnote}

Reporting Checklist: The authors have completed the STROBE reporting checklist. Available at https://dx.doi. org/10.21037/apm-21-1019

Data Sharing Statement: Available at https://dx.doi. org/10.21037/apm-21-1019

Conflicts of Interest: All authors have completed the ICMJE uniform disclosure form (available at https://dx.doi. org/10.21037/apm-21-1019). The authors have no conflicts of interest to declare.

Ethical Statement: The authors are accountable for all aspects of the work in ensuring that questions related to the accuracy or integrity of any part of the work are appropriately investigated and resolved. The study was conducted in accordance with the Declaration of Helsinki (as revised in 2013) and was approved by the Renji Hospital Ethics Committee of Shanghai Jiaotong University School of Medicine (No. 2014-148K and 2016-142K). All patients gave their informed consent prior to their inclusion in the study.

Open Access Statement: This is an Open Access article distributed in accordance with the Creative Commons Attribution-NonCommercial-NoDerivs 4.0 International
License (CC BY-NC-ND 4.0), which permits the noncommercial replication and distribution of the article with the strict proviso that no changes or edits are made and the original work is properly cited (including links to both the formal publication through the relevant DOI and the license). See: https://creativecommons.org/licenses/by-nc-nd/4.0/.

\section{References}

1. GBD 2017 Disease and Injury Incidence and Prevalence Collaborators. Global, regional, and national incidence, prevalence, and years lived with disability for 354 diseases and injuries for 195 countries and territories, 1990-2017: a systematic analysis for the Global Burden of Disease Study 2017. Lancet 2018;392:1789-858.

2. GBD 2017 Causes of Death Collaborators. Global, regional, and national age-sex-specific mortality for 282 causes of death in 195 countries and territories, 1980-2017: a systematic analysis for the Global Burden of Disease Study 2017. Lancet 2018;392:1736-88.

3. Zhou M, Wang H, Zeng X, et al. Mortality, morbidity, and risk factors in China and its provinces, 1990-2017: a systematic analysis for the Global Burden of Disease Study 2017. Lancet 2019;394:1145-58.

4. Sarin SK, Kumar M, Eslam M, et al. Liver diseases in the Asia-Pacific region: a Lancet Gastroenterology \& Hepatology Commission. Lancet Gastroenterol Hepatol 2020;5:167-228.

5. Qiao L, Wang X, Deng G, et al. Cohort profile: a multicentre prospective validation cohort of the Chinese Acute-on-Chronic Liver Failure (CATCH-LIFE) study. BMJ Open 2021;11:e037793.

6. Hu CL, Du QC, Wang ZX, et al. Relationship between platelet-based models and the prognosis of patients with malignant hepatic tumors. Oncol Lett 2020;19:2384-96.

7. Malehmir M, Pfister D, Gallage S, et al. Platelet GPIb $\alpha$ is a mediator and potential interventional target for NASH and subsequent liver cancer. Nat Med 2019;25:641-55.

8. Kurokawa T, Zheng YW, Ohkohchi N. Novel functions of platelets in the liver. J Gastroenterol Hepatol 2016;31:745-51.

9. Afdhal NH, Giannini EG, Tayyab G, et al. Eltrombopag before procedures in patients with cirrhosis and thrombocytopenia. N Engl J Med 2012;367:716-24.

10. Vincent JL, Moreno R, Takala J, et al. The SOFA (Sepsisrelated Organ Failure Assessment) score to describe organ dysfunction/failure. On behalf of the Working Group on Sepsis-Related Problems of the European 
Society of Intensive Care Medicine. Intensive Care Med 1996;22:707-10.

11. Moreau R, Jalan R, Gines P, et al. Acute-on-chronic liver failure is a distinct syndrome that develops in patients with acute decompensation of cirrhosis. Gastroenterology 2013;144:1426-37, 1437.e1-9.

12. Vallet-Pichard A, Mallet V, Nalpas B, et al. FIB-4: an inexpensive and accurate marker of fibrosis in $\mathrm{HCV}$ infection. comparison with liver biopsy and fibrotest. Hepatology 2007;46:32-6.

13. Wai CT, Greenson JK, Fontana RJ, et al. A simple noninvasive index can predict both significant fibrosis and cirrhosis in patients with chronic hepatitis C. Hepatology 2003;38:518-26.

14. de Franchis R; Baveno VI Faculty. Expanding consensus in portal hypertension: Report of the Baveno VI Consensus Workshop: Stratifying risk and individualizing care for portal hypertension. J Hepatol 2015;63:743-52.

15. Fan R, Papatheodoridis G, Sun J, et al. aMAP risk score predicts hepatocellular carcinoma development in patients with chronic hepatitis. J Hepatol 2020;73:1368-78.

16. Gu WY, Xu BY, Zheng X, et al. Acute-on-Chronic Liver Failure in China: Rationale for Developing a Patient Registry and Baseline Characteristics. Am J Epidemiol 2018;187:1829-39.

17. Zhang Y, Xu BY, Wang XB, et al. Prevalence and Clinical Significance of Portal Vein Thrombosis in Patients With Cirrhosis and Acute Decompensation. Clin Gastroenterol Hepatol 2020;18:2564-2572.e1.

18. AlEssa HB, Bhupathiraju SN, Malik VS, et al. Carbohydrate quality and quantity and risk of type 2 diabetes in US women. Am J Clin Nutr 2015;102:1543-53.

19. Spaite DW, Hu C, Bobrow BJ, et al. Mortality and Prehospital Blood Pressure in Patients With Major Traumatic Brain Injury: Implications for the Hypotension Threshold. JAMA Surg 2017;152:360-8.

20. Terrault NA, Hassanein T, Howell CD, et al. Phase II study of avatrombopag in thrombocytopenic patients with cirrhosis undergoing an elective procedure. J Hepatol

Cite this article as: Ouyang $\mathrm{R}, \mathrm{Li} \mathrm{H}$, Xia J, Wang X, Zheng X, Huang Y, Meng Z, Gao Y, Qian Z, Liu F, Lu X, Shi Y, Shang J, Liu J, Deng G, Zheng Y, Yan H, Zhang W, Qiao L, Jiang X, Wang H, Zhong G, Li B, Chen J. Lower platelet counts were associated with 90-day adverse outcomes in acute-on-chronic liver disease patients. Ann Palliat Med 2021;10(9):9342-9353. doi: 10.21037/apm-21-1019
2014;61:1253-9.

21. Afdhal N, McHutchison J, Brown R, et al. Thrombocytopenia associated with chronic liver disease. J Hepatol 2008;48:1000-7.

22. Wehler M, Kokoska J, Reulbach U, et al. Short-term prognosis in critically ill patients with cirrhosis assessed by prognostic scoring systems. Hepatology 2001;34:255-61.

23. Trebicka J, Fernandez J, Papp M, et al. The PREDICT study uncovers three clinical courses of acutely decompensated cirrhosis that have distinct pathophysiology. J Hepatol 2020;73:842-54.

24. Wang B, Zhou J, Wu X, et al. Screening varices in patients with HBV-related cirrhosis on antiviral therapy: Platelet alone or together with LSM. Liver Int 2021;41:369-77.

25. Giannini EG. Review article: thrombocytopenia in chronic liver disease and pharmacologic treatment options. Aliment Pharmacol Ther 2006;23:1055-65.

26. Hermos JA, Altincatal A, Weber HC, et al. Thrombocytopenia and bleeding in veterans with nonhepatitis C-related chronic liver disease. Dig Dis Sci 2013;58:562-73.

27. Peck-Radosavljevic M, Simon K, Iacobellis A, et al. Lusutrombopag for the Treatment of Thrombocytopenia in Patients With Chronic Liver Disease Undergoing Invasive Procedures (L-PLUS 2). Hepatology 2019;70:1336-48.

28. Kawaguchi T, Komori A, Seike M, et al. Efficacy and safety of eltrombopag in Japanese patients with chronic liver disease and thrombocytopenia: a randomized, openlabel, phase II study. J Gastroenterol 2012;47:1342-51.

29. Stravitz RT, Ellerbe C, Durkalski V, et al. Thrombocytopenia Is Associated With Multi-organ System Failure in Patients With Acute Liver Failure. Clin Gastroenterol Hepatol 2016;14:613-620.e4.

30. Xu X, Hou Z, Xu Y, et al. The dynamic of platelet count as a novel and valuable predictor for 90-day survival of hepatitis B virus-related acute-on-chronic liver failure patients. Clin Res Hepatol Gastroenterol 2021;45:101482. 


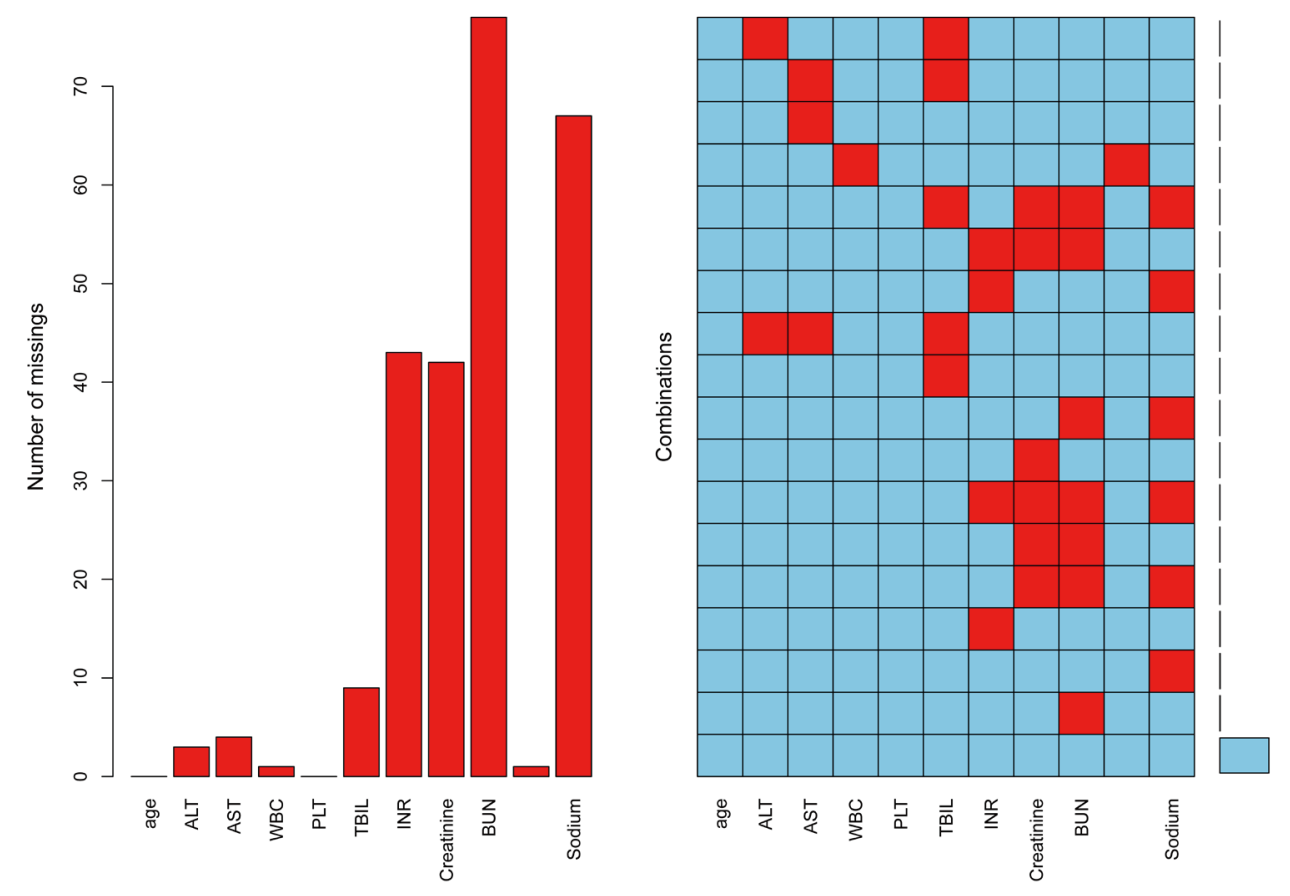

Figure S1 Missing value distribution.

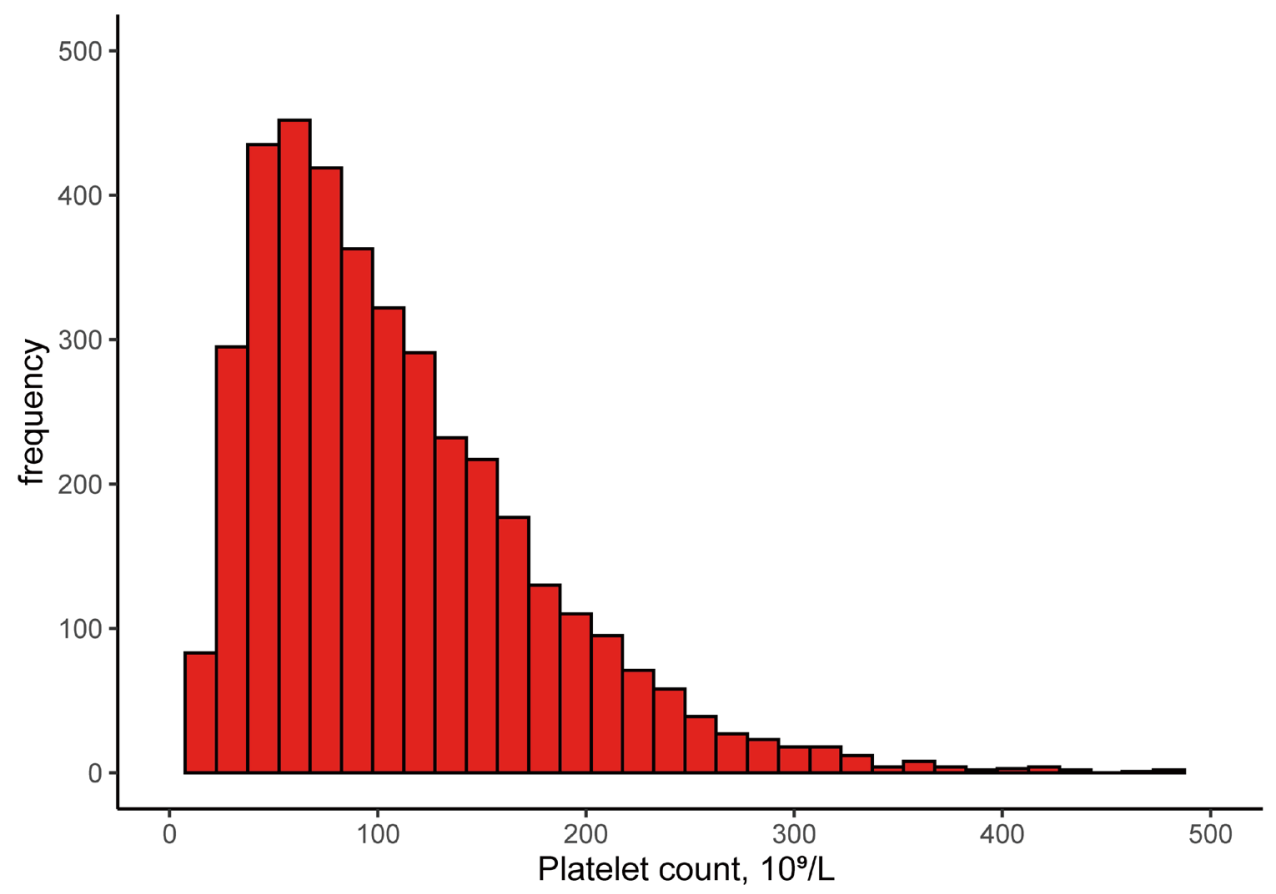

Figure S2 Platelet count frequency. 


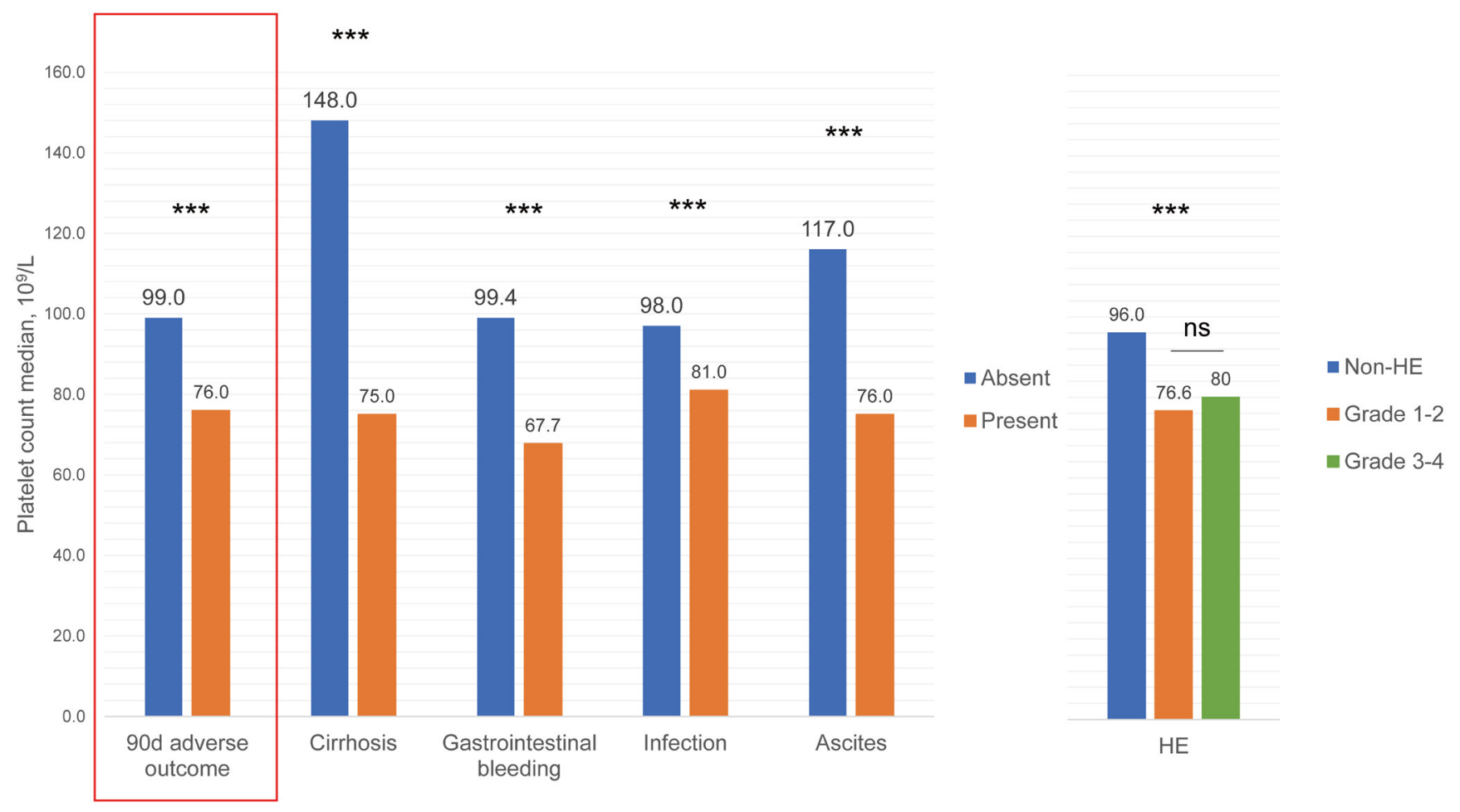

${ }^{* * *} p<0.001$, ns: non-significant

Figure S3 Median Platelet count across acute decompensation subgroups. 
A

Platelet count, 10\%/L $-<20-20-50-50-100-100-150-\geq 150$

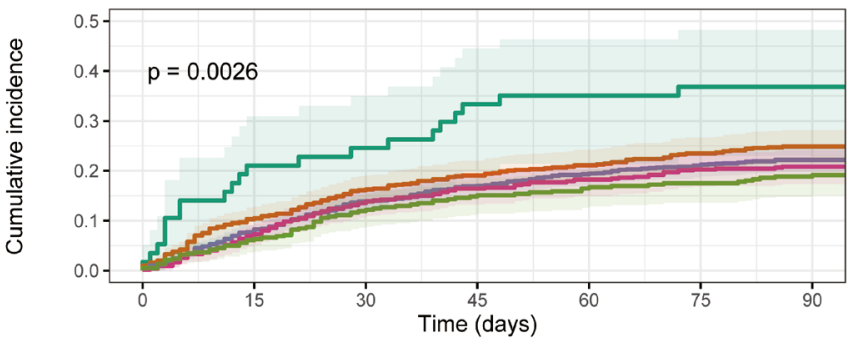

Number at risk

\begin{tabular}{|r|ccccccc|}
$<20$ & 57 & 45 & 43 & 38 & 37 & 36 & 36 \\
$20-50$ & 640 & 573 & 536 & 517 & 504 & 489 & 476 \\
$50-100$ & 1179 & 1088 & 1015 & 977 & 949 & 927 & 912 \\
$100-150$ & 548 & 510 & 473 & 457 & 447 & 436 & 432 \\
$\geq 150$ & 378 & 355 & 332 & 319 & 311 & 307 & 302 \\
\cline { 2 - 8 } & & & & & & &
\end{tabular}

C Platelet count, $10^{9} / \mathrm{L}-<20-20-50-50-100-100-150-\geq 150$

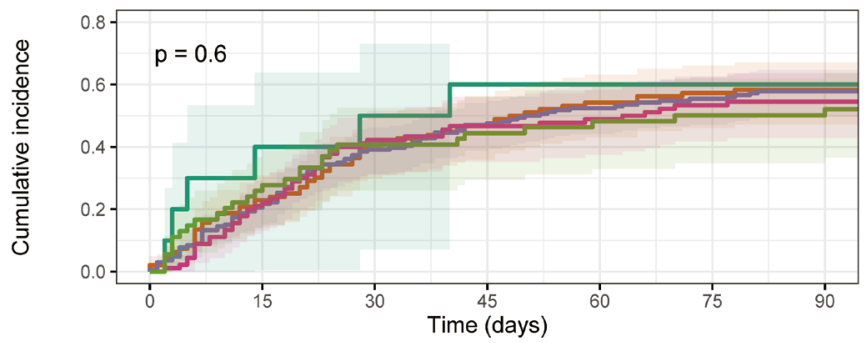

Number at risk

\begin{tabular}{|r|ccccccc|}
\cline { 2 - 8 } $20-50$ & 10 & 6 & 5 & 4 & 4 & 4 & 4 \\
$50-100$ & 166 & 74 & 57 & 51 & 44 & 41 & 39 \\
$100-150$ & 90 & 132 & 101 & 88 & 79 & 74 & 68 \\
$\geq 150$ & 54 & 71 & 52 & 48 & 46 & 42 & 40 \\
\cline { 2 - 9 } & & 40 & 32 & 30 & 27 & 26 & 26 \\
\hline
\end{tabular}

B

Platelet count, 10\%/L $\quad-$ 20-50 $-50-100-100-150-\geq 150$

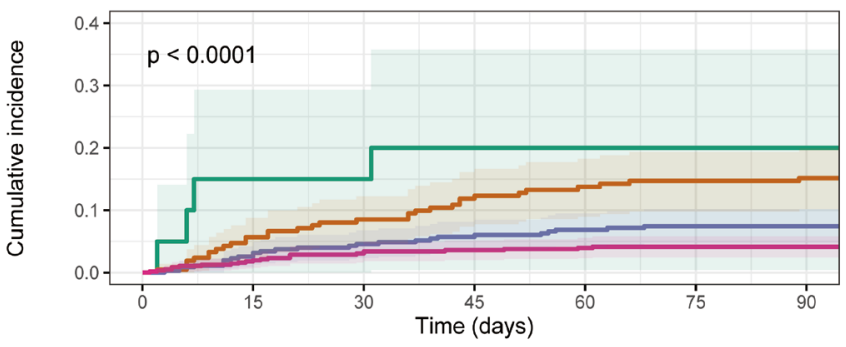

Number at risk

\begin{tabular}{|c|c|c|c|c|c|c|c|}
\hline $20-50$ & 20 & 17 & 17 & 16 & 16 & 16 & 16 \\
\hline $50-100$ & 211 & 199 & 193 & 186 & 182 & 180 & 178 \\
\hline $100-150$ & 349 & 340 & 333 & 329 & 325 & 323 & 322 \\
\hline$\geq 150$ & 557 & 547 & 539 & 536 & 534 & 533 & 532 \\
\hline
\end{tabular}

D Platelet count, $10 \%$ L $-<20-20-50-50-100-100-150-\geq 150$

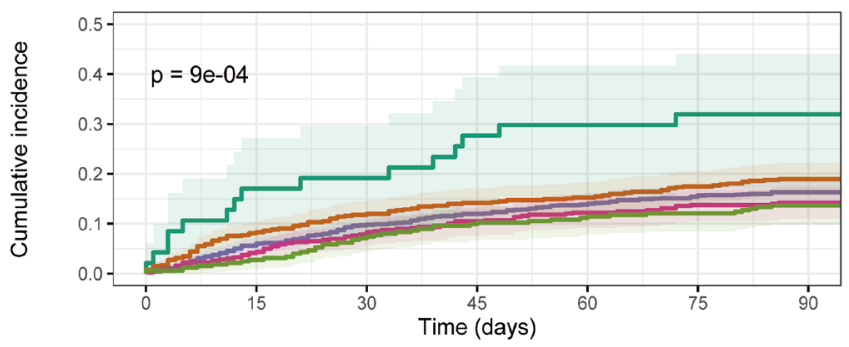

Number at risk

\begin{tabular}{r|ccccccc|}
\cline { 2 - 8 }$<20$ & 47 & 39 & 38 & 34 & 33 & 32 & 32 \\
$20-50$ & 544 & 499 & 479 & 466 & 460 & 448 & 437 \\
$50-100$ & 1013 & 956 & 914 & 889 & 870 & 853 & 844 \\
$100-150$ & 458 & 439 & 421 & 409 & 401 & 394 & 392 \\
$\geq 150$ & 324 & 315 & 300 & 289 & 284 & 281 & 276 \\
\cline { 2 - 8 } & & & & & & &
\end{tabular}

Figure S4 Kaplan-Meier graph of 90-day adverse outcome. A Patients with cirrhosis. B Patients without cirrhosis. C Patients with ACLF. D Patients without ACLF. 
Table S1 Univariate and multivariate analysis of 90-day adverse outcome (Patients with ACLF and non-ACLF)

\begin{tabular}{|c|c|c|c|c|}
\hline \multirow{2}{*}{ Variables, $10^{9} / \mathrm{L}$} & \multirow{2}{*}{$\begin{array}{l}\text { 90-day adverse outcome, } \\
\text { n (\%) }\end{array}$} & \multirow{2}{*}{$\begin{array}{l}\text { PLT median, } \\
10^{9} / \mathrm{L}\end{array}$} & Unadjusted model & Model $3^{a}$ \\
\hline & & & \multicolumn{2}{|c|}{ Odds ratio (95\% Confidence interval) } \\
\hline ACLF patients & $243(58.4)$ & 80.0 & & \\
\hline PLT<20 & $6(60.0)$ & 15.0 & $1.29(0.33-5.11)$ & \\
\hline $20 \leq \mathrm{PLT}<50$ & $57(59.4)$ & 36.0 & $1.26(0.64-2.47)$ & \\
\hline $100 \leq \mathrm{PLT}<150$ & $53(58.9)$ & 119.5 & $1.23(0.63-2.44)$ & \\
\hline PLT $\geq 150$ & 29(53.7) & 170.5 & 1 [Reference] & \\
\hline$P$ value for trend ${ }^{b}$ & & & 0.540 & \\
\hline $\mathrm{PLT}<20$ & 15(31.9) & 15.0 & $2.98(1.50-5.95)$ & $3.35(1.55-7.25)$ \\
\hline $20 \leq \mathrm{PLT}<50$ & 105(19.3) & 38.0 & $1.52(1.04-2.23)$ & $1.83(1.16-2.86)$ \\
\hline $50 \leq \mathrm{PLT}<100$ & 168(16.6) & 70.0 & $1.27(0.88-1.81)$ & $1.37(0.91-2.06)$ \\
\hline $100 \leq \mathrm{PLT}<150$ & $66(14.4)$ & 119.5 & $1.07(0.71-1.62)$ & $1.12(0.71-1.77)$ \\
\hline$P L T \geq 150$ & $44(13.6)$ & 196.0 & 1 [Reference] & 1 [Reference] \\
\hline$P$ value for trend ${ }^{b}$ & & & 0.005 & 0.002 \\
\hline PLT (Continuous- pe & crease) & & $1.01(0.99-1.03)$ & $1.01(0.99-1.03)$ \\
\hline
\end{tabular}

Abbreviations: ACLF, acute-on-chronic liver failure; PLT, platelet count; ALT, aspartate aminotransferase; WBC, white blood cell; INR, international normalized ratio; $A D$, acute decompensation; HE, hepatic encephalopathy; Model 1: adjusted for age, sex, cirrhosis, and etiology; Model 2: adjusted for model 1 plus laboratory variables (ALT, WBC, hemoglobin, sodium, total bilirubin, INR, and creatinine); ${ }^{a}$ Model 3: adjusted for model 2 plus AD (gastrointestinal bleeding, infection, ascites, and HE); ${ }^{\mathrm{b}}$ Test for trend based on variable containing median value for each group. 
Table S2 Univariate and multivariate sensitivity analysis of 90-day adverse outcome

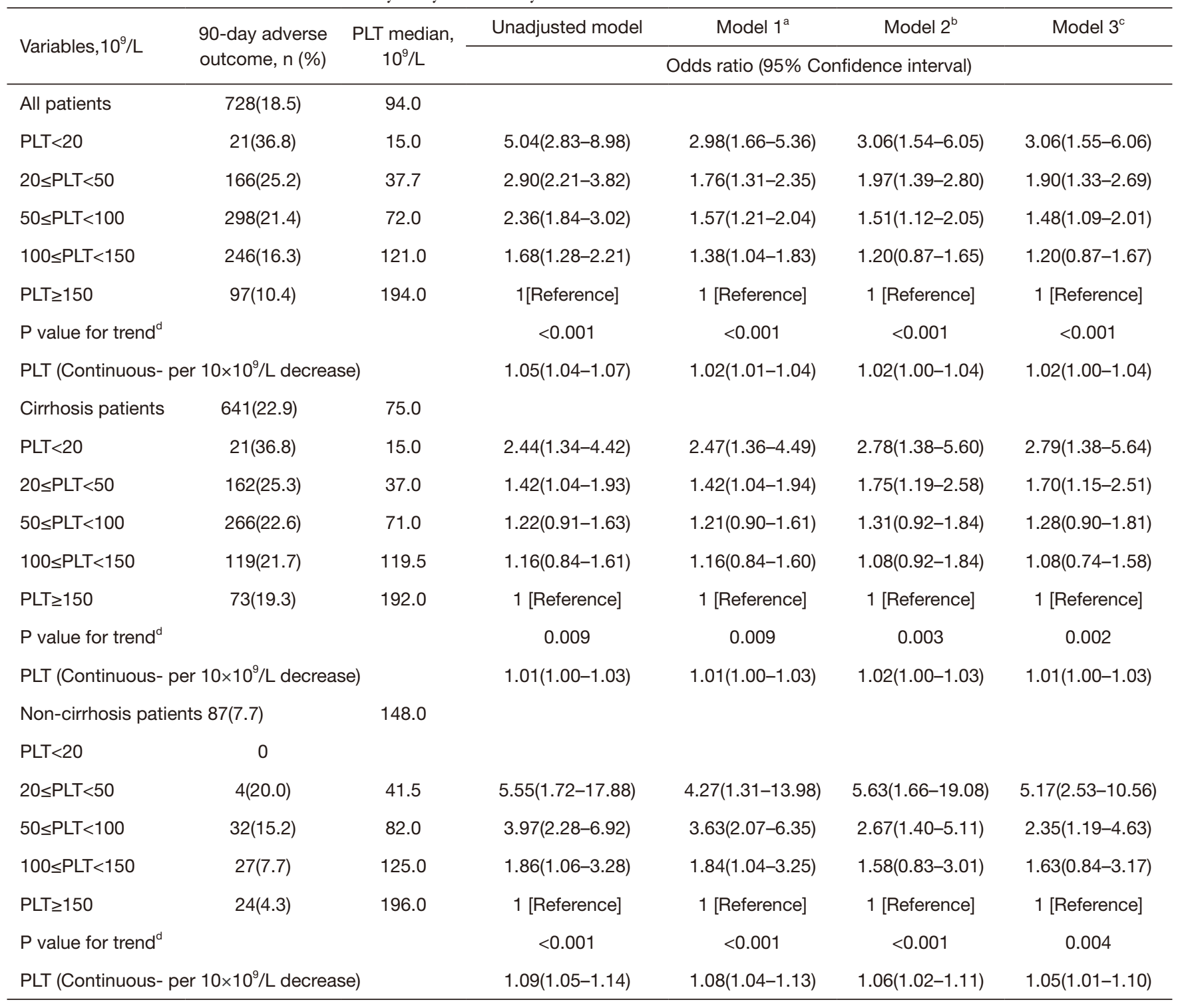

Abbreviations: PLT, platelet count; ALT, aspartate aminotransferase; WBC, white blood cell; INR, international normalized ratio; AD, acute decompensation; HE, hepatic encephalopathy; ${ }^{a}$ Model 1: adjusted for age, sex, cirrhosis, and etiology; ${ }^{b}$ Model 2: adjusted for model 1 plus laboratory variables (ALT, WBC, hemoglobin, sodium, total bilirubin, INR, and creatinine); ${ }^{c}$ Model 3: adjusted for model 2 plus AD (gastrointestinal bleeding, infection, ascites, and HE); ${ }^{d}$ Test for trend based on variable containing median value for each group 


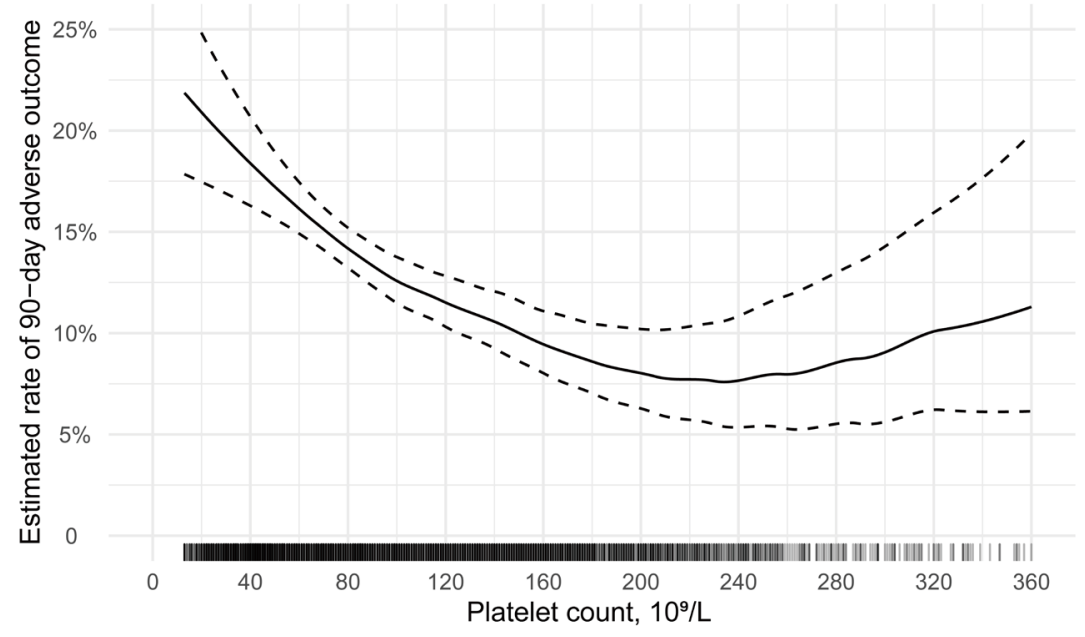

Figure S5 Association of platelet count and the incidence of 90-day adverse outcome in adjusted model 3; Model 1: adjusted for age, sex, cirrhosis, and etiology; Model 2: adjusted for model 1 plus laboratory variables (ALT, WBC, hemoglobin, sodium, total bilirubin, INR, and creatinine); Model 3: adjusted for model 2 plus AD (gastrointestinal bleeding, infection, ascites, and HE). 